\title{
Wnt signaling pathway upregulates DNMT1 to trigger NHERF1 promoter hypermethylation in colon cancer
}

\author{
YONGLI GUO*, MENG WANG ${ }^{*}$, XUEFENG JIA*, HUA ZHU, YAQIN ZHI and LEILEI YUAN \\ Department of Oncology, Jining No. 1 People's Hospital, Jining, Shandong 272051, P.R. China
}

Received December 21, 2017; Accepted May 31, 2018

DOI: $10.3892 / o r .2018 .6494$

\begin{abstract}
NHERF1 is downregulated and has been identified as a new marker of colorectal cancer. However, the molecular mechanism of NHERF1 downregulation in colon cancer is not well understood. In the present study, we demonstrated that the NHERF1 mRNA level was downregulated and correlated with outcomes in colon cancer patients. NHERF1 expression was associated with EMT phenotype. High levels of promoter methylation of NHERF1 in colon cancer were observed. NHERF1 expression was restored by demethylation treatment. In addition, a negative correlation between methylation and mRNA expression of NHERF1 was observed in the TCGA dataset. Moreover, DNMT1 inhibited NHERF1 expression in vivo. DNMT1 expression was found to be negatively correlated with NHERF1 and NHERF1 expression was also restored by a DNMT1 inhibitor. DNMT1 and NHERF1 were regulated by the Wnt signaling pathway. In addition, among DNMT1 high expression patients, the Wnt signaling pathway was negatively correlated with NHERF1 expression. In contrast, among DNMT1 low expression patients, the Wnt signaling pathway was not correlated with NHERF1 expression. In conclusion, Wnt signaling increases DNMT1 expression. DNMT1 contributes to promoter hypermethylation and epigenetic NHERF1 silencing in colon cancer. In addition, we provide novel insight into the mechanisms underlying the regulation of NHERF1 expression and occurrence/progression of colon cancer.
\end{abstract}

Correspondence to: Mrs. Leilei Yuan, Department of Oncology, Jining No. 1 People's Hospital, 6 Xingfu Road, Jining, Shandong 272051, P.R. China

E-mail: yl10994@sina.com

${ }^{*}$ Contributed equally

Abbreviations: NHERF1, $\mathrm{Na}^{+} / \mathrm{H}^{+}$exchanger regulatory factor 1 ; CA, adenocarcinoma of the colon; DNMT1, DNA methyltransferase 1; EMT, epithelial-to-mesenchymal transition; 6-TG, 6-thioguanine

Key words: NHERF1, DNMT1, methylation, Wnt signaling, colon cancer

\section{Introduction}

The Wnt signaling pathway controls various cellular events such as migration, proliferation and differentiation throughout development (1). Deregulation of Wnt signaling has been found in colon cancer where, most frequently, mutations in APC lead to cytoplasmic accumulation of $\beta$-catenin and its translocation to the nucleus $(2,3)$. Following translocation to the nucleus, $\beta$-catenin binds to T-cell factor/lymphoid enhancer factor (TCF/LEF), which activates transcription of target genes and ultimately results in the uncontrolled cell proliferation of tumor cells (4). Although nuclear $\beta$-catenin expression level is a biomarker associated with invasion, metastasis and poor prognosis of colon cancer, it cannot explain the phenotypic heterogeneity of colon cancer.

$\mathrm{Na}^{+} / \mathrm{H}^{+}$exchanger regulatory factor 1 (NHERF1/EBP50), an adaptor molecule that interacts with $\beta$-catenin, has been recently implicated in the progression of colon cancer $(5,6)$. This complex regulates Wnt signaling and may cooperate in the development of colon cancer. Other influences that enhance the activation of the $\mathrm{Wnt} / \beta$-catenin pathway have been documented, either from components of the Wnt pathway itself or from parallel pathways that connect to the Wnt pathway, such as the PI3K/PTEN/Akt pathway (7) or the Hippo-YAP/TAZ pathway (8). Notably, NHERF1 also interacts with Frizzled, PTEN and YAP (6) and suppresses the Wnt signaling pathway, the PI3K/PTEN/Akt pathway and the Hippo-YAP/TAZ pathway, respectively. Moreover, there is some evidence for a dual role of NHERF1 in cancer, depending on its intracellular localization. However, NHERF1 $^{-/}$immortalized mouse embryonic fibroblasts (MEFs), in contrast to their wild-type counterparts, present anchorage-independent growth and increased migration (5). Decreased survival and increased intestinal tumor burden were observed in $\mathrm{Apc}^{\mathrm{Min} / \mathrm{N}} \mathrm{NHERF} 1^{-/}$double-mutant mice (6). In addition, during the steps involved in early colon carcinogenesis, NHERF1 is gradually lost at the expense of nuclear and cytoplasmic expression in adenoma and is a new marker of colorectal cancer (9). As a whole, NHERF1 appears to behave as a tumor suppressor in colon cancer. However, the molecular mechanism of NHERF1 downregulation in colon cancer is not well understood.

In the present study, we revealed that NHERF1 was significantly downregulated in colon adenocarcinoma (CA) tissues and was correlated with patient outcomes. Meanwhile, 
NHERF1 expression was associated with epithelial-to-mesenchymal transition (EMT) phenotype in CA cells. A predicted $\mathrm{CpG}$ island was located within the NHERF1 promoter and NHERF1 was found to be silenced by promoter methylation in CA. DNA methyltransferase 1 (DNMT1) is one of the key transmethylases leading to the hypermethylation status of the NHERF1 promoter and silencing of NHERF1 in CA. Inhibition of the Wnt signaling pathway upregulated NHERF1 and significantly downregulated DNMT1 expression. In addition, regulation of NHERF1 expression was found to be dependent on DNMT1 expression in CA. These results have elucidated the molecular mechanisms underlying the downregulation of NHERF1 and occurrence/progression of CA.

\section{Materials and methods}

The Cancer Genome Atlas (TCGA) data. The mRNA data (RNA Seq v2) and clinical information for patients in the TCGA_COAD dataset were downloaded from https://www. synapse.org and cBioPortal database (www.cbioportal.org), respectively and used for differential mRNA expression, correlation, prognosis and gene set enrichment analysis.

GEO dataset collection and differential expression analysis. Microarray data were obtained from 4 datasets. The 4 series were accessed at the National Centers for Biotechnology Information (NCBI) Gene Expression Omnibus (GEO) database (http://www.ncbi.nlm.nih.gov/geo/) which served as a public repository for gene expression datasets, and the accession numbers were GSE21510, GSE32323, GSE91370, GSE79740, GSE77955, GSE60620, GSE18560 and GSE97023. Differentially expressed genes were obtained using GEO2R (http://www.ncbi.nlm.nih.gov/geo/geo2r/). GEO2R is an interactive web tool that compares two groups of samples under the same experimental conditions and can analyze almost any GEO series (10).

Gene set enrichment analysis. The association between phenotypes, pathways and NHERF1 expression was analyzed using Gene Set Enrichment Analysis (GSEA v2.2, http://www.broad. mit.edu/gsea/). GSEA calculates a gene set Enrichment Score (ES) that estimates whether genes from pre-defined gene set [obtained from the Molecular Signatures Database, MSigDB, http://software.broadinstitute.org,POSITIVE_REGULATION_OF _EPITHELIAL_TO_MESENCHYMAL_TRANSITION, $\mathrm{H}$ ALLMARK_WNT_BETA_CATENIN_SIGNALING] are enriched among the highest- (or lowest-) ranked genes or distributed randomly. Default settings were used. Thresholds for significance were determined by permutation analysis (1,000 permutations). False discovery rate (FDR) was calculated. A gene set was considered significantly enriched when the FDR score was $<0.05$.

The IHC data in colon cancer. The IHC-based NHERF1 protein expression data including high-resolution images were viewed and downloaded from The Human Protein Atlas web portal (www.proteinatlas.org). ImageJ (National Institutes of Health, Bethesda, MD, USA) was used to evaluate the NHERF1 expression and localization.
Cell culture. Human colon cancer cell lines were purchased from the Cell Resource Center of Beijing Xiehe (Beijing, China) and cultivated in an incubator at $37^{\circ} \mathrm{C}$ with $5 \% \mathrm{CO}_{2}$. HCT116, SW480, LoVo and HT29 cells were maintained in high-glucose Dulbecco's modified Eagle's medium (DMEM; Gibco; Thermo Fisher Scientific, Inc., Waltham, MA, USA) supplemented with HyClone ${ }^{\mathrm{TM}}$ 10\% fetal bovine serum (FBS; GE Healthcare Life Sciences, Logan, UT, USA) as well as penicillin (100 U/ml; Thermo Fisher Scientific).

Methylation-specific PCR. The CpG island of the NHERF1 gene was predicted by Database of CpG Islands (http://dbcat. cgm.ntu.edu.tw/). Genomic DNA was extracted for methylation analysis from cells in culture by using the Genomic DNA Miniprep Kit (Sigma-Aldrich; Merck KGaA) One microgram of genomic DNA was modified with sodium bisulfite using the DNA Bisulfite Conversion kit (Tiangen Biotech, Co., Ltd., Beijing, China) according to the specifications of the manufacturer. Methylation-specific PCR (MSP) was run in a total volume of $20 \mu \mathrm{l}$. MSP reactions were subjected to initial incubation at $95^{\circ} \mathrm{C}$ for $5 \mathrm{~min}$, followed by 35 cycles of $94^{\circ} \mathrm{C}$ for $20 \mathrm{sec}$, and annealing at $60^{\circ} \mathrm{C}$ for $30 \mathrm{sec}$ and $72^{\circ} \mathrm{C}$ for $20 \mathrm{sec}$. Final extension was conducted by incubation at $72^{\circ} \mathrm{C}$ for $5 \mathrm{~min}$. MSP products were separated on $2 \%$ agarose gels and visualized after ethidium bromide staining. The following primers were used: Unmethylated forward, 5'-TAGTTTAGTTTG GGTGATAGAGTGA-3' and unmethylated reverse, 5'-CAA CCTAATTTCCCCTACCTATAACA-3'; methylated forward, 5'-GTAGTTTAGTTTGGGCGATAGAGC-3' and methylated reverse, 5'-GACCTAATTTCCCCTACCTATAACG-3'.

RNA isolation and real-time quantitative PCR analyses. Total RNA was isolated using TRIzol (Sigma-Aldrich; Merck KGaA) and cDNAs were synthesized using a Reverse Transcription kit (cat. no. 1708842; Bio-Rad Laboratories, Hercules, CA, USA). Real-time quantitative RT-PCR was performed using Applied Biosystems ${ }^{\circledR}$ SYBR-Green Master Mix on the 7900HT Fast Real-Time PCR System (Thermo Fisher Scientific).

Demethylation treatment. Cells were treated with $2 \mu \mathrm{M}$ 5-aza-2'-deoxycytidine or $3 \mu \mathrm{M} 6$-thioguanine (Sigma-Aldrich; Merck KGaA) for 3-4 days, with medium and 5-aza-2'-deoxycytidine replenished every day. After treatment, the cells were collected for RNA isolation.

Western blotting. Samples were run on $10 \%$ sodium dodecyl sulfate (SDS)-polyacrylamide gels (PAGE) and transferred to NC membranes. The blots were blocked in blocking buffer (5\% non-fat dry milk in TBST buffer) for $1 \mathrm{~h}$ at room temperature and then incubated with primary antibodies $(1: 1,000)$ in blocking buffer overnight at $4^{\circ} \mathrm{C}$. The blots were washed 3 times with TBST buffer and incubated for $1 \mathrm{~h}$ at room temperature with a horseradish peroxidase (HRP)-conjugated anti-mouse IgG (cat. no. A4416) or anti-rabbit IgG (cat. no. A4914) secondary antibody (1:3,000; Sigma-Aldrich; Merck KGaA, Darmstadt, Germany) in blocking buffer. Finally, the blots were washed 3 times with TBST buffer and visualized via enzyme-linked chemiluminescence using the electrochemiluminescence (ECL) kit (Applygen Technologies Inc., Beijing, China). 
A
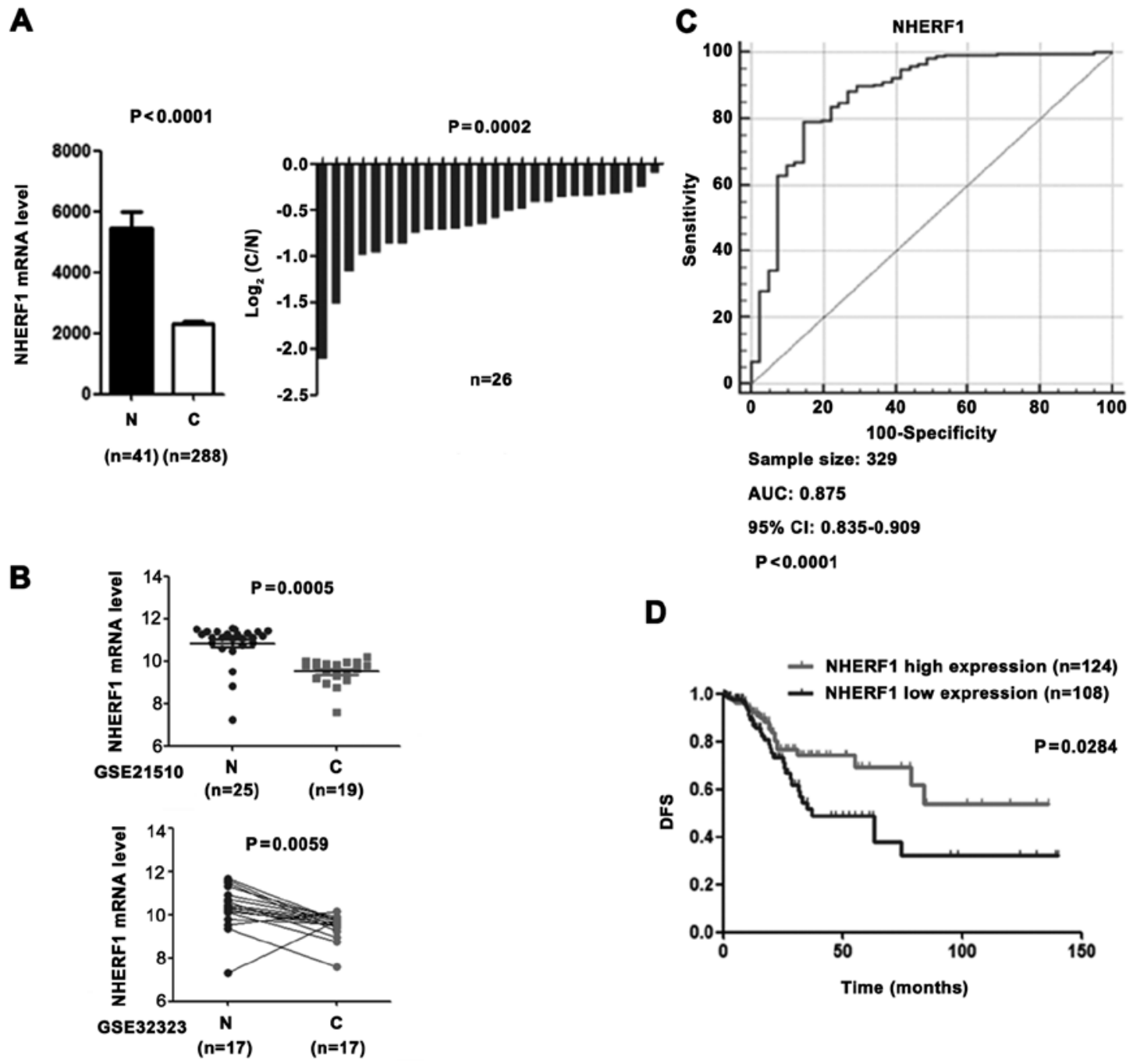

D

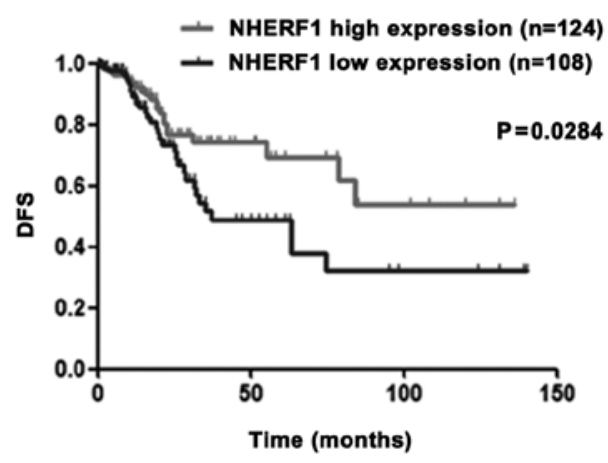

Figure 1. The low expression level of NHERF1 in CA is associated with a poor prognosis. (A) NHERF1 mRNA level in CA (C) and adjacent normal tissues $(\mathrm{N})$ from the TCGA COAD dataset. Left panel, bar graph shows NHERF1 expression in CA $(\mathrm{n}=288)$ and adjacent normal tissues $(\mathrm{n}=41$, independent sample t-test, $\mathrm{P}<0.0001)$. Error bar represent standard error of the mean (SEM). Right panel, relative NHERF1 expression in CA patients $(\mathrm{n}=26)$ and their matched normal tissues ( $\mathrm{n}=26$, paired sample t-test, $\mathrm{P}=0.0002$ ). (B) NHERF1 expression in CA tissues from independent validation sets. GSE21510: 19 CA tissues and 25 normal controls (independent sample t-test, $\mathrm{P}=0.0005$ ). GSE32323: $17 \mathrm{CA}$ tissues and 17 paired adjacent normal tissues (paired sample t-test, $\mathrm{P}=0.0059$ ). (C) NHERF1 showed ROC curves and an AUC with diagnostic power to distinguish CA patients from healthy controls. The area under the ROC curve was 0.875 (95\% CI, 0.835-0.909). (D) KM curve for DFS of patients with relative high $(\mathrm{n}=124)$ and low $(\mathrm{n}=108)$ NHERF1 mRNA level. The curve indicated a shorter DFS time $(\mathrm{P}<0.05)$ with low NHERF1 mRNA level. CA, colon adenocarcinoma; ROC, receiver operating characteristic curve; AUC, area under the ROC curve; KM, Kaplan-Meier; DFS, disease-free survival; CI, confidence interval.

The primary antibody specific for NHERF1 (cat. no. 611160) was purchased from BD Biosciences (San Jose, CA, USA), the primary antibodies specific for GAPDH (cat. no. 5174) was purchased from Cell Signaling Technology, Inc. (Danvers, MA, USA).

Cancer cell line Encyclopedia database. The data concerning APC and $\beta$-catenin mutation in colorectal cancer cell lines were obtained from the Cancer Cell Line Encyclopedia (https://portals.broadinstitute.org/ccle).

Statistical analysis. Statistical analyses were performed using SPSS 19.0 (IBM Corp., Armonk, NY, USA) and GraphPad Prism 5 (GraphPad Software, Inc., San Diego, CA, USA). Group distributions were compared using the Student's t-test. A value of $\mathrm{P}<0.05$ was considered to indicate a statistically significant result. The correlation of NHERF1 and
EMT marker expression levels were analyzed by Pearson's correlation analysis.

\section{Results}

NHERF1 mRNA expression is downregulated in CA tissues and is correlated with patient outcomes. To investigate the role of NHERF1 in CA patients, we compared the NHERF1 expression level in adjacent normal tissues vs. tumour tissues using the colon adenocarcinoma (COAD) mRNASeq v2 data set in The Cancer Genome Atlas (TCGA) and revealed that NHERF1 was significantly downregulated in CA tissues (Fig. 1A). Consistent with the above results, downregulation of NHERF1 in CA was validated from 2 independent GEO datasets (Fig. 1B; GSE21510 and GSE32323). In addition, NHERF1 expression was also able to significantly distinguish CA tissues and adjacent normal tissues, revealing 
A

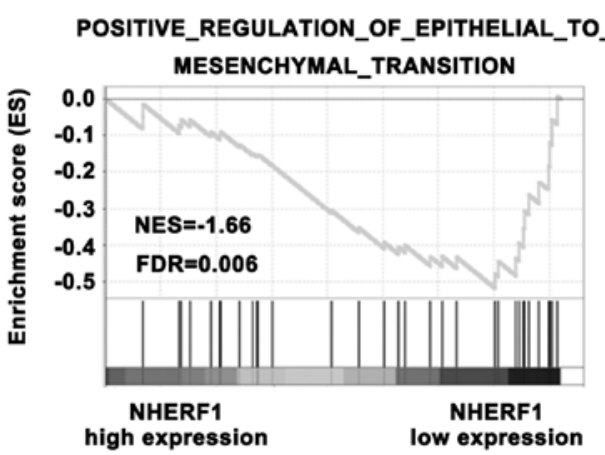

C

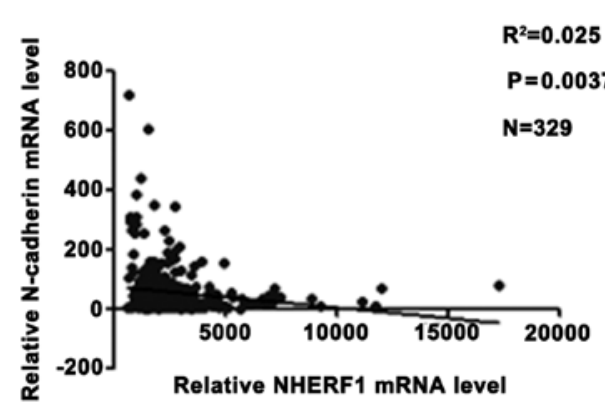

$\mathbf{E}$

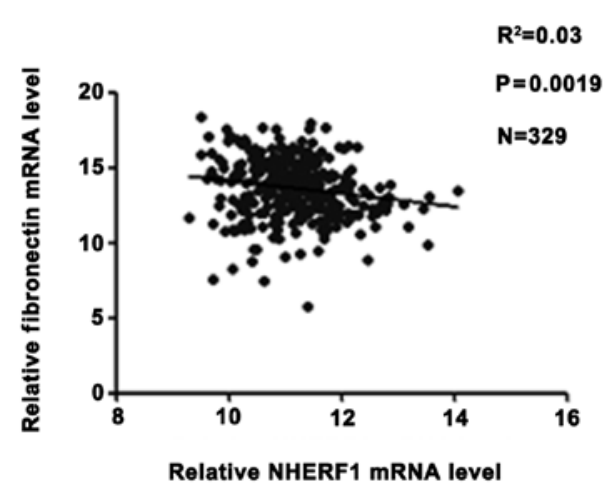

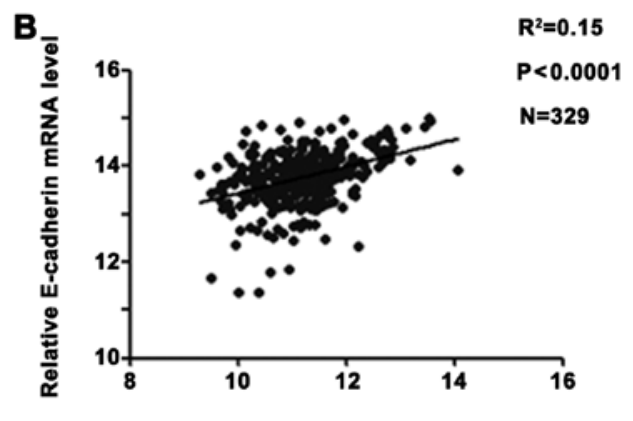

Relative NHERF1 mRNA level

D

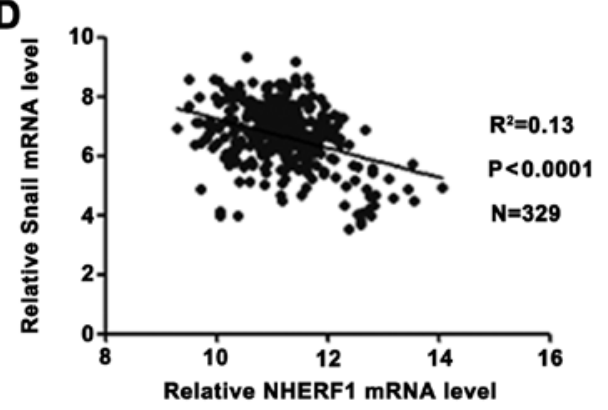

$\mathbf{F}$

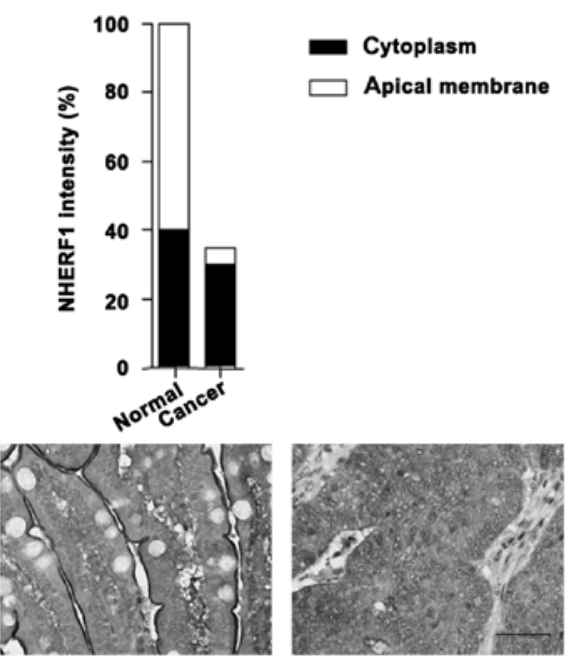

Figure 2. NHERF1 expression is correlated with EMT status in CA tissues. (A) Enrichment plot of gene expression signatures for EMT according to NHERF1 mRNA levels by gene set enrichment analysis (GSEA) of TCGA dataset. Samples were divided into high and low NHERF1 expression groups. False discovery rate (FDR) provides the estimated probability that a gene set with a given normalized ES (NES) represents a false-positive finding; FDR $<0.05$ is a widely accepted cut-off for the identification of biologically significant gene sets. (B-E) Correlation between NHERF1 and expression of EMT markers in CA. NHERF1 expression level was negatively correlated with the expression level of epithelial marker E-cadherin $\left(B ; R^{2}=0.15, P<0.0001\right)$ and positively correlated with expression levels of mesenchymal markers: $\mathrm{N}$-cadherin $\left(C ; R^{2}=0.025, P=0.0037\right)$, Snail $\left(D ; R^{2}=0.13, P<0.0001\right)$ and fibronectin $\left(E ; R^{2}=0.03, P=0.0019\right)$ in CA tissues ( $\mathrm{n}=329)$. (F) The Human Protein Atlas database results revealed that the expression level of NHERF1 was downregulated in colon cancer tissues compared with normal tissues. In normal tissue, NHERF1 was mainly located in the apical membrane. In contrast, NHERF1 was mainly located in the cytoplasm in colon cancer tissue. Scale bar, $50 \mu \mathrm{m}$. CA, colon adenocarcinoma; EMT, epithelial-to-mesenchymal transition.

that NHERF1 has the advantage for acting as a prognostic marker in CA (Fig. 1C). To further analyze the prognostic value of NHERF1 in CA tissues, the Kaplan-Meier analyses was conducted on the TCGA_COAD dataset. As shown in Fig. 1D, low NHERF1 expression was found to be significantly associated with worse disease-free survival (DFS) (log-rank $\mathrm{P}=0.0284)$ of CA patients. These results demonstrated that CA tissues had low NHERF1 expression and that patients with low NHERF1 expression had poor prognoses. Our findings suggest that NHERF1 may play an important role in CA occurrence and progression in patients in the clinical setting.
Low NHERF1 expression is associated with EMT phenotype in $C A$. In a previous study, NHERF1 depletion induced EMT-like changes in a colorectal cancer cell line (9). In order to further determine the role of NHERF1 in clinical patients, we performed additional analyses on TCGA_COAD dataset. GSEA result revealed that the NHERF1 expression level was negatively correlated with the EMT phenotype (Fig. 2A) and levels of mesenchymal markers ( $\mathrm{N}$-cadherin, Snail and fibronectin), but was positively correlated with the level of epithelial marker E-cadherin in CA tissues (Fig. 2B-E), suggesting a close correlation between the NHERF1 level and 
A DataBase of CpG islands and Analytical Tool (DBCAT) $1000 \mathrm{bp}$
Possible CpG islands shown in graph:

CPG island region CPG site The most dense CPG island

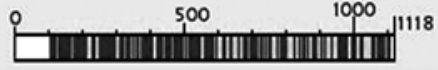

Sequence of each CpG island:

1. start site: 106 end site: 1108 length:1003 GC content:67\%

B

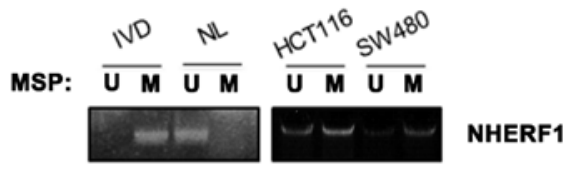

D

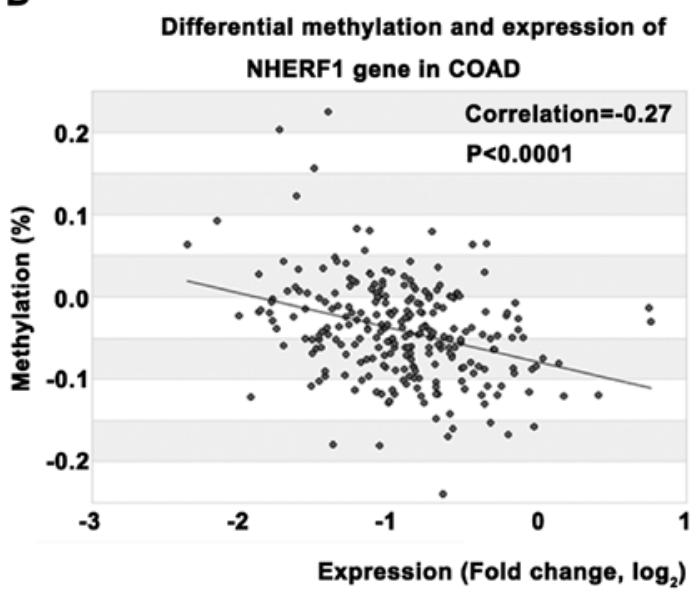

C

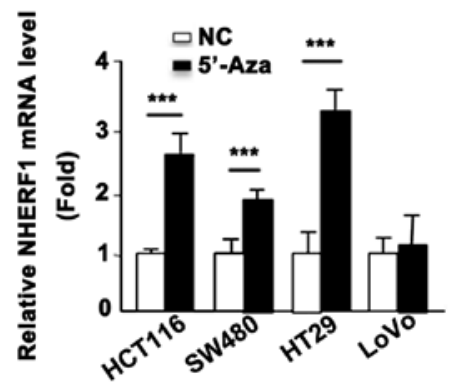

E

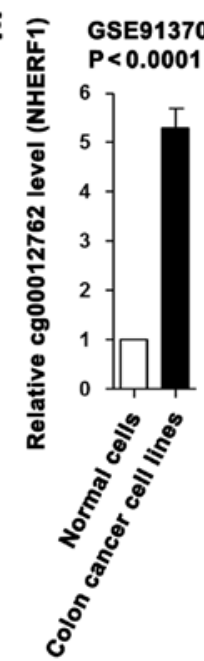

DNA sequence input

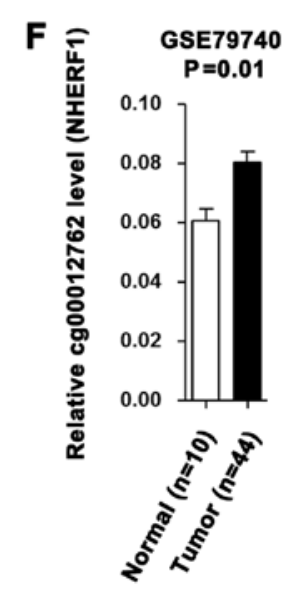

Figure 3. NHERF1 is silenced by promoter hypermethylation in CA. (A) The Database of CpG islands identified a CpG island within the NHERF1 gene promoter region (upstream 1,000 bp of NHERF1). Black line depicts the $\mathrm{CpG}$ island region and the gray vertical bars represent a CpG site. White line depicts the DNA sequence. (B) Dense DNA methylation level of the NHERF1 gene promoter region was indicated in colon cancer cell lines by MSP. The methylation status of NHERF1 in HCT116 and SW480 cells was determined by MSP. Primer efficiency was verified by a positive control (IVD) and negative control (NL). (C) NHERF1 expression was analyzed by RT-PCR in colon cancer cell lines before and after 5-Aza treatment. Cells were treated with $5 \mathrm{mM} 5$-Aza for $72 \mathrm{~h}$, and the medium was replaced with freshly added 5-Aza for every $24 \mathrm{~h}\left({ }^{* * * *} \mathrm{P}<0.0001\right)$. (D) A negative correlation between methylation percentage and mRNA levels of NHERF1 in the TCGA cohort of COAD samples. The line shows the linear regression of methylation percentage and mRNA expression $\left(\log _{2}\right)$. (E) The methylation level of NHERF1 in cell lines from GEO database (GSE91370). (F) The methylation level of NHERF1 in colon cancer and normal tissues from GEO database (GSE79740). CA, colon adenocarcinoma; MSP, methylation-specific PCR; IVD, in vitro methylated DNA; NL, normal blood lymphocyte DNA; U, unmethylated alleles; M, methylated alleles; 5-Aza, 5-azacytidine.

EMT phenotype. These results suggest that NHERF1 possibly acts as a potential tumor suppressor in CA by inhibiting the EMT process. In addition, we also found that the apical membrane NHERF1 was significantly reduced in tumor tissues, implying that apical membrane NHERF1 expression was negatively correlated with EMT and the malignant phenotype in colon cancer patients (Fig. 2F). These findings revealed the clinical role of NHERF1 in colon cancer.

NHERF1 is silenced by promoter methylation in CA. High promoter methylation level always leads to transcriptional gene silencing. Sequence analysis using Database of $\mathrm{CpG}$ islands (http://dbcat.cgm.ntu.edu.tw/) revealed that a $\mathrm{CpG}$ island (or CpG-rich sequence) is located within the NHERF1 promoter (Fig. 3A). Next, we performed MSP assays to detect the DNA methylation status of the NHERF1 promoter in CA cell lines. As shown in Fig. 3B, DNA was hypermethylated in the HCT116 and SW480 cells. Furthermore, demethylation treatment by 5-aza-2-deoxycytidine (5'-Aza) successfully restored the mRNA level of NHERF1 in the colon cancer cell lines (Fig. 3C). To further evaluate the correlation between methylation and transcription of NHERF1 in CA, we retrieved methylation and mRNA expression data on NHERF1 from the MethHC database (http://methhc.mbc.nctu.edu.tw/php/index. php). Linear regression analysis demonstrated a significant negative correlation between mRNA and promoter methylation levels of NHERF1 (Fig. 3D). In addition, high levels of promoter methylation of NHERF1 in colon cancer cell 
A

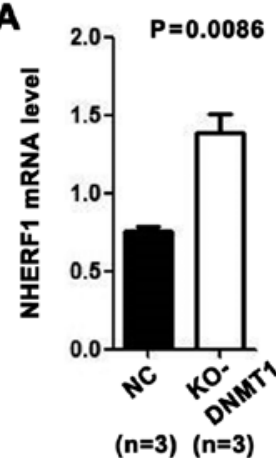

D

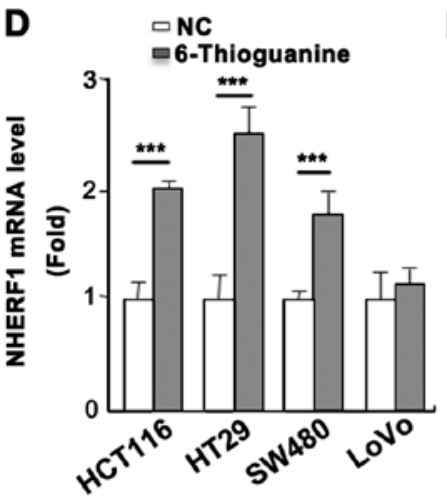

E

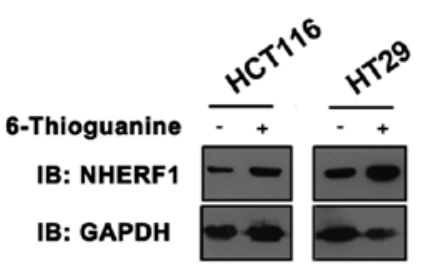

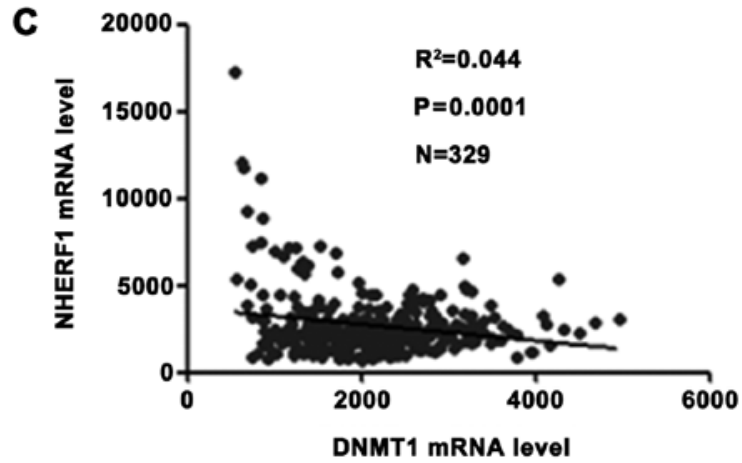

$\mathbf{F}$

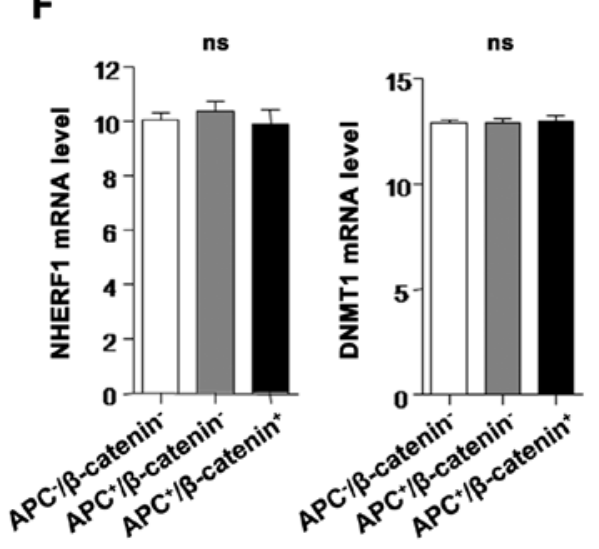

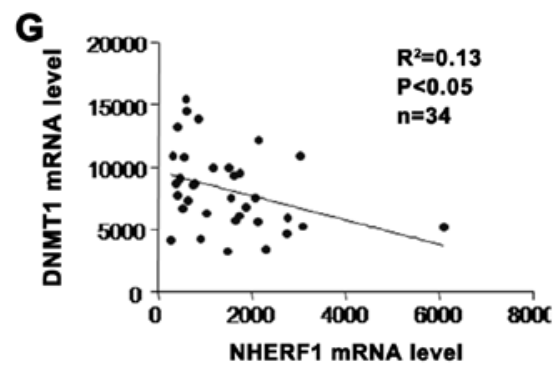

Figure 4. NHERF1 was reduced by transmethylase DNMT1 in CA. (A) NHERF1 expression was upregulated in DNMT1-knockout (KO) mice comparing with negative controls (GSE60620, $\mathrm{n}=3$, paired sample $\mathrm{t}$-test, $\mathrm{P}=0.0086$ ). (B) DNMT1 mRNA level in CA and adjacent normal tissues (N) from the TCGA_COAD dataset. Bar graph shows DNMT1 expression in CA $(C)(n=288)$ and adjacent normal tissues $(N)(n=41$, independent sample $t$-test, $P<0.0001)$. (C) Correlation between NHERF1 and DNMT1 expression in CA ( $\mathrm{n}=329 ; \mathrm{R} 2=0.044, \mathrm{P}=0.0001)$ in CA tissues from the TCGA_COAD dataset. (D) 6-Thioguanine (6-TG) treatment resulted in increased NHERF1 mRNA level in colon cancer cell lines. The NHERF1 expression was analyzed by RT-PCR before and after 6-TG treatment $\left({ }^{* * *} \mathrm{P}<0.0001\right)$. (E) 6-TG treatment resulted in increased NHERF1 protein level in colon cancer cell lines. Cell lysates were detected by western blotting with anti-NHERF1. GAPDH served as a loading control. (F) NHERF1 (left) and DNMT1 (right) expression in the non-mutated group (APC/ $\beta$-catenin; $\mathrm{n}=20$ ), APC-mutated group ( $\mathrm{APC}^{+} / \beta$-catenin; $\mathrm{n}=10$ ) and the $\mathrm{APC} / \beta$-catenin-mutated group $\left(\mathrm{APC}^{+} / \beta\right.$-catenin $\left.{ }^{+} ; \mathrm{n}=3\right)$. ns, not significant. (G) Correlation between NHERF1 and DNMT1 expression in colon cancer cell lines $(n=34 ; R 2=0.13, P<0.05)$. CA, colon adenocarcinoma.

lines, and low methylation in normal epithelial cells were noted (Fig. 3E). The promoter methylation level of NHERF1 was also higher in colon cancer tissues than the level noted in the normal tissues (Fig. 3F). These findings demonstrated that NHERF1 is downregulated by promoter hypermethylation in CA.

DNMT1 expression correlates with low NHERF1 mRNA level in $C A$. With the highest reduction of DNMT1 in colon cancer cells, extensive loss of DNA methylation occurred in the promoter and hypermethylated $\mathrm{CpG}$ islands, indicating a major role for DNMT1 in maintaining genomic DNA methylation (11). A high DNMT1 expression level in tumors suggests a causal link to DNA hypermethylation and silenced NHERF1 expression. To test this hypothesis, we looked at the association between DNMT1 expression and the NHERF1
mRNA level from GEO and TCGA databases. As shown in Fig. 4A, the NHERF1 mRNA level was upregulated in DNMT1-knockout (KO) mice. To further evaluate the correlation between DNMT1 and NHERF1, TCGA_COAD dataset was used. We revealed that the expression of DNMT1 was significantly upregulated and was negatively correlated with the expression of NHERF1 in CA tissues (Fig. 4B and C). To further investigate whether NHERF1 expression is reduced by DNMT1, the DNMT1 inhibitor 6-thioguanine (6-TG), causing downregulation of DNMT1 through ubiquitin-targeted degradation (12), was used. As shown in Fig. 4D, the mRNA levels of NHERF1 were significantly upregulated in HCT116, HT29 and SW480 cells treated with 6-TG. However, NHERF1 mRNA did not change in the LoVo cells treated with 6-TG. In addition, the protein levels of NHERF1 were consistent with the mRNA levels in the HCT116 and HT29 cells (Fig. 4E). It 


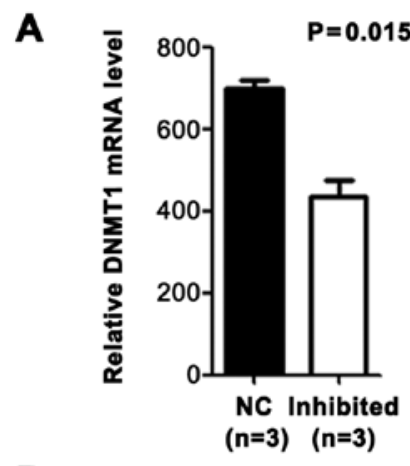

B HALLMARK_WNT_BETA_CATENIN_SIGNALING (DNMT1 high expression)

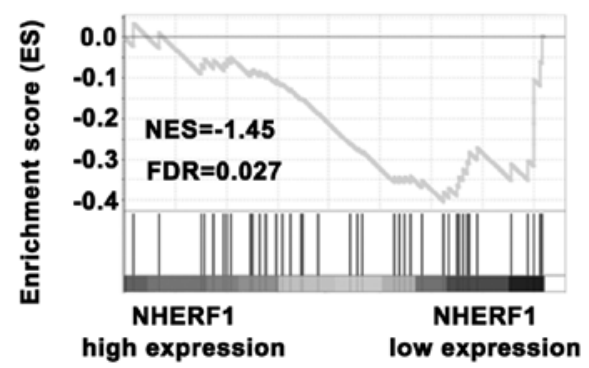

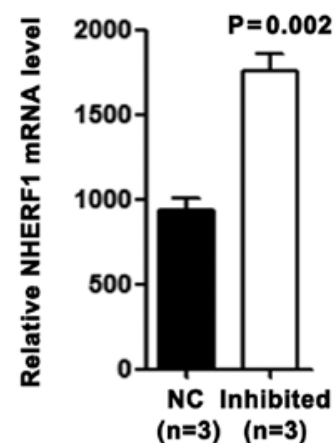

HALLMARK_WNT_BETA_CATENIN_SIGNALING

(DNMT1 low expression)

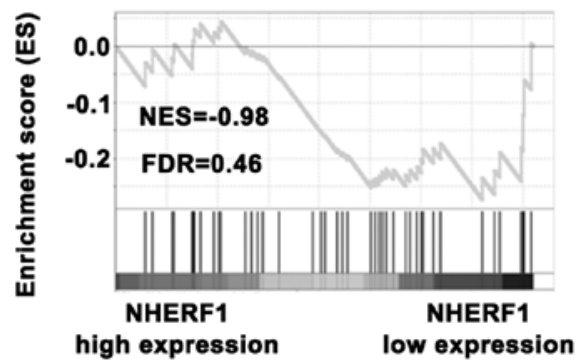

Figure 5. Wnt/ $\beta$-catenin signaling inhibits NHERF1 mRNA expression via increasing DNMT1. (A) DNMT1 and NHERF1 expression in response to Wnt signaling inhibition in colon cancer cells (GSE18560). Left panel, bar graph shows DNMT1 expression in the Wnt signaling inhibition group (Inhibited) and negative control group (NC) ( $\mathrm{n}=3$, paired sample t-test, $\mathrm{P}=0.015)$. Right panel, NHERF1 expression in the Wnt signaling inhibition group (Inhibited) and negative control group $(\mathrm{NC})(\mathrm{n}=3$, paired sample t-test, $\mathrm{P}=0.002)$. (B) Enrichment plots of gene expression signatures for Wnt signaling according to NHERF1 mRNA levels by gene set enrichment analysis (GSEA) of TCGA_COAD dataset in the DNMT1 high expression group (NES=-1.45, FDR <0.05) and DNMT1 low (with almost undetectable DNMT1 level) expression group (NES=-0.98, FDR=0.46), respectively. Samples were divided into high and low NHERF1 expression groups. False discovery rate (FDR) provides the estimated probability that a gene set with a given normalized enrichment score (NES) represents a false-positive finding; FDR $<0.05$ is a widely accepted cut-off for the identification of biologically significant gene sets.

is known that APC and $\beta$-catenin are usually mutated in colon cancer cell lines and are involved in colon cancer progression. To detect the regulatory effect of DNMT1 on NHERF1 in different mutated cell lines, GEO database was used. As shown in Fig. 4F, the expression of NHERF1 and DNMT1 were not significantly different in the non-mutated group, the APC-mutated group and the APC/ $\beta$-catenin-mutated group. In addition, NHERF1 was negatively correlated with DNMT1 expression in 34 colon cancer cell lines (Fig. 4G). These results suggest that DNMT1 may inhibit NHERF1 expression in most colon cancer cell lines and this effect is not related with APC or $\beta$-catenin mutations.

Wnt signaling pathway promotes DNMT1 expression and inhibits NHERF1 expression in CA. Colorectal cancer arises from activating mutations in the Wnt signaling pathway that converge with additional molecular changes to shape tumor development and patient prognosis (9). We demonstrated that DNMT1 was upregulated in CA tissues and caused the downregulation of NHERF1. To investigate whether it is associated with overactivated Wnt signaling pathway in CA, the GEO database was used. As shown in Fig. 5A, the DNMT1 mRNA level was significantly downregulated and the NHERF1 mRNA level was significantly upregulated in colon cancer cell line Ls174T after Wnt signaling inhibition (GSE18560). To further evaluate the relationship between the Wnt signaling pathway and these 2 genes, we performed GSEA. As shown in Fig. 5B, among DNMT1 high expression CA patients, the Wnt signaling pathway activation gene set was highly enriched in the NHERF1 low expression group. On the contrary, among DNMT1 low expression CA patients, Wnt signaling pathway activation gene set was not correlated with NHERF1 expression. These results suggest that the overactivated Wnt signaling pathway promotes DNMT1 expression and may inhibit NHERF1 expression in a DNMT1-dependent manner in CA.

\section{Discussion}

The scaffold protein NHERF1/EBP50 contains an ERMbinding region and two PDZ domains. NHERF1 always exerts its function via association with multiple partners and by regulating multiple signaling pathways, such as the Wnt signaling pathway $(1,5,13)$, the PI3K/PTEN/Akt pathway $(7,14,15)$, the Hippo-YAP/TAZ pathway (8) and parathyroid hormone 1 receptor signalling (16). In $\mathrm{NHERF}^{-/-}$ mice, Ezrin-radixin-moesin (ERM) proteins are significantly reduced in brush border membranes from kidney and small intestine (17). In addition, NHERF1 ${ }^{-/-}$females were generally smaller and showed impaired mobility consistent with muscle weakness that was apparent in several performance coordination tests. Most of the small NHERF $1^{-/-}$female animals died 30-35 days after birth. Autopsies of dead NHERF1 ${ }^{-/-}$females suggested that some developed hydrocephaly (18).

Recently, NHERF1 has been implicated in the progression of various human malignancies. NHERF1 gene mutations found with low frequency (3\%) in breast cancer cell lines and primary tumors were associated with loss of heterozygosity and 
increased aggressiveness (19), suggesting a tumor-suppressor role for NHERF1. NHERF1 ${ }^{-/}$immortalized mouse embryonic fibroblasts (MEFs), in contrast to their wild-type counterparts, presented anchorage-independent growth and increased migration (5), supportive of a tumor-suppressor function for NHERF1. NHERF1 acts as a tumor suppressor in vivo for intestinal adenoma development via suppressing the Wnt signalling pathway. Decreased survival and increased intestinal tumor burden were observed in $\mathrm{Apc}^{\mathrm{Min} /+} \mathrm{NHERF}^{-1-}$ double-mutant mice (6). In contrast, Shibata et al (20) reported that the NHERF $1 / \beta$-catenin complex promotes Wnt signaling and may cooperate in the development of liver cancer. There has been increasing evidence for a dual role of NHERF1 in cancer, depending on its intracellular localization. In a previous study, apical membrane NHERF1 depletion induced EMT-like changes and cytoplasmic NHERF1 promoted cell proliferation in colon cancer cells (9). In addition, nuclear NHERF1 is involved during colon cancer carcinogenesis (21-23). In fact, phosphorylation has been shown to regulate assembly and disassembly of cellular target complexes, NHERF1 subcellular localization and oligomerization. In addition, it has been shown that NHERF1 phosphorylation status depends on the cell cycle and also plays a role in its progression (24-26). Although NHERF1 is tightly controlled in cells, NHERF1 appears to behave as a tumor suppressor in various human malignancies.

It was reported that NHERF1 is significantly downregulated in multiple cancers, including colorectal cancer (9), particularly in early carcinogenesis (21), cervical (27), pancreatic cancer (28) and high-grade glioblastoma (14). Recently, NHERF1 is regarded as a new marker in colorectal cancer (9). In the present study, our results indicated that NHERF1 is downregulated in colon adenocarcinoma which is consistent with previous reports. Although the clinical role of NHERF1 in colon cancer is somewhat clear, the mechanism underlying the downregulation of NHERF1 is not well understood. In previous studies, the NHERF1 promoter contains 13 half-estrogen-responsive elements and NHERF1 regulation by estrogens has been evidenced in several carcinoma cell types including endometrial, mammary (29) and biliary epithelial cells (30). In vitro, positive regulation of NHERF1 by estrogens was illustrated in ER-positive breast carcinoma cell lines; whereas no NHERF1 stimulation was noted in ER-negative ones (31-33). In addition, there is no direct evidence, but several studies have suggested that NHERF1 may potentially be subjected to epigenetic modifications (24).

The present study is the first direct demonstration that NHERF1 expression is regulated by epigenetic modifications in cancer. It is known that global DNA hypomethylation is a general characteristic of cancer (34) and the progression of DNA hypomethylation may be driven by 'oncogenes' (35). However, genome-wide studies of cancer epigenomes reveal that $1-10 \%$ of $\mathrm{CpG}$ islands are aberrantly methylated, which suggests that thousands of gene promoters may be hypermethylated in the average cancer (36). The aberrant hypermethylation of genes appears to be a common molecular mechanism for silencing tumor-suppressor genes and can contribute to cancer formation through the transcriptional repression of these genes $(37,38)$. We found that NHERF1 was downregulated by promoter hypermethylation in colon cancer.
Activated Wnt signaling-mediated upregulation of DNMT1 led to a hypermethylation status of the NHERF1 promoter and silenced NHERF1 expression in colon adenocarcinoma. In addition, activated Wnt signaling appears to inhibit NHERF1 expression in a DNMT1- dependent manner. These results suggest that activated Wnt signaling by mutations in APC inhibits NHERF1 expression by promoting DNMT1 expression and loss of NHERF1 overactivated Wnt signaling pathway in colon adenocarcinoma, identifying a feedback loop in the Wnt/DNMT1/NHERF1 pathway. In addition, we also observed that NHERF1 is correlated with the EMT phenotype which is associated with poor prognosis in colon adenocarcinoma patients. Collectively, the data reported in this study demonstrated, for the first time, a novel relationship between the Wnt signaling pathway and NHERF1. NHERF1 did not only inhibit the Wnt signaling pathway but was also downregulated by the Wnt signaling pathway in colon adenocarcinoma.

In summary, our data may provide a new insight in mechanism of NHERF1 downregualtion in early occurrence steps of carcinogenesis, suggesting that Wnt/DNMT1/NHERF1 axis acts as a crucial role in occurrence and progression of colon cancer.

\section{Acknowledgements}

The authors thank Dr Z. Peng (State Key Laboratory of Proteomics, Beijing Proteome Research Center, Beijing Institute of Radiation Medicine, Collaborative Innovation Center for Cancer Medicine, Beijing, China) for kindly providing materials and technical assistance.

\section{Funding}

No funding was received.

\section{Availability of data and materials}

The datasets used during the present study are available from the corresponding author upon reasonable request.

\section{Authors' contributions}

LY conceived and designed the experiments; YG performed the experiments, MW analyzed the data and XJ wrote the paper; $\mathrm{HZ}$ and $\mathrm{YZ}$ contributed by procuring the reagents, materials and analytical tools. In addition, HZ performed TCGA data and IHC analysis and YZ performed GSEA and methylation data analysis. All authors read and approved the manuscript and agree to be accountable for all aspects of the research in ensuring that the accuracy or integrity of any part of the work are appropriately investigated and resolved.

\section{Ethics approval and consent to participate}

Not applicable.

\section{Patient consent for publication}

Not applicable. 


\section{Competing interests}

The authors state that they have no competing interests.

\section{References}

1. Lin YY, Hsu YH, Huang HY, Shann YJ, Huang CY, Wei SC, Chen CL and Jou TS: Aberrant nuclear localization of EBP50 promotes colorectal carcinogenesis in xenotransplanted mice by modulating TCF-1 and $\beta$-catenin interactions. J Clin Invest 122 : 1881-1894, 2012.

2. Giles RH, van Es JH and Clevers H: Caught up in a wht storm: Wnt signaling in cancer. Biochim Biophys Acta 1653: 1-24, 2003

3. Wangefjord S, Brandstedt J, Lindquist KE, Nodin B, Jirstrom K and Eberhard J: Associations of beta-catenin alterations and MSI screening status with expression of key cell cycle regulating proteins and survival from colorectal cancer. Diagn Pathol 8: 10, 2013.

4. Stanczak A, Stec R, Bodnar L, Olszewski W, Cichowicz M, Kozlowski W, Szczylik C, Pietrucha T, Wieczorek M and Lamparska-Przybysz M: Prognostic significance of Wnt-1, $\beta$-catenin and E-cadherin expression in advanced colorectal carcinoma. Pathol Oncol Res 17: 955-963, 2011.

5. Kreimann EL, Morales FC, de Orbeta-Cruz J, Takahashi Y Adams H, Liu TJ, McCrea PD and Georgescu MM: Cortical stabilization of beta-catenin contributes to NHERF1/EBP50 tumor suppressor function. Oncogene 26: 5290-5299, 2007.

6. Georgescu MM, Gagea M and Cote G: NHERF1/EBP50 suppresses wnt- $\beta$-catenin pathway-driven intestinal neoplasia. Neoplasia 18: 512-523, 2016.

7. Parsons DW, Wang TL, Samuels Y, Bardelli A, Cummins JM, DeLong L, Silliman N, Ptak J, Szabo S, Willson JK, et al: Colorectal cancer: Mutations in a signalling pathway. Nature 436: 792, 2005.

8. Azzolin L, Panciera T, Soligo S, Enzo E, Bicciato S, Dupont S, Bresolin S, Frasson C, Basso G, Guzzardo V, et al: YAP/TAZ incorporation in the $\beta$-catenin destruction complex orchestrates the Wnt response. Cell 158: 157-170, 2014.

9. Hayashi Y, Molina JR, Hamilton SR and Georgescu MM: NHERF1/EBP50 is a new marker in colorectal cancer Neoplasia 12: 1013-1022, 2010

10. Barrett T, Wilhite SE, Ledoux P, Evangelista C, Kim IF, Tomashevsky M, Marshall KA, Phillippy KH, Sherman PM, Holko M, et al: NCBI GEO: Archive for functional genomics data sets-update. Nucleic Acids Res 41: D991-D995, 2013.

11. Cai Y, Tsai HC, Yen RC, Zhang YW, Kong X, Wang W, Xia L and Baylin SB: Critical threshold levels of DNA methyltransferase 1 are required to maintain DNA methylation across the genome in human cancer cells. Genome Res 27: 533-544, 2017.

12. Flesner BK, Kumar SR and Bryan JN: 6-Thioguanine and zebularine down-regulate DNMT1 and globally demethylate canine malignant lymphoid cells. BMC Vet Res 10: 290, 2014.

13. WheelerDS,Barrick SR, Grubisha MJ,Brufsky AM,FriedmanPA and Romero G: Direct interaction between NHERF1 and Frizzled regulates $\beta$-catenin signaling. Oncogene 30: 32-42, 2011.

14. Molina JR, Agarwal NK, Morales FC, Hayashi Y, Aldape KD, Cote $\mathrm{G}$ and Georgescu MM: PTEN, NHERF1 and PHLPP form a tumor suppressor network that is disabled in glioblastoma. Oncogene 31: 1264-1274, 2012.

15. Molina JR, Morales FC, Hayashi Y, Aldape KD and Georgescu MM: Loss of PTEN binding adapter protein NHERF1 from plasma membrane in glioblastoma contributes to PTEN inactivation. Cancer Res 70: 6697-6703, 2010.

16. Mahon MJ, Donowitz M, Yun CC and Segre GV: $\mathrm{Na}^{+} / \mathrm{H}^{+}$ exchanger regulatory factor 2 directs parathyroid hormone 1 receptor signalling. Nature 417: 858-861, 2002.

17. Morales FC, Takahashi Y, Kreimann EL and Georgescu MM: Ezrin-radixin-moesin (ERM)-binding phosphoprotein 50 organizes ERM proteins at the apical membrane of polarized epithelia. Proc Natl Acad Sci USA 101: 17705-17710, 2004.

18. Shenolikar S, Voltz JW, Minkoff CM, Wade JB and Weinman EJ Targeted disruption of the mouse NHERF-1 gene promotes internalization of proximal tubule sodium-phosphate cotransporter type IIa and renal phosphate wasting. Proc Natl Acad Sci USA 99: 11470-11475, 2002.

19. Dai JL, Wang L, Sahin AA, Broemeling LD, Schutte M and Pan Y: NHERF $\left(\mathrm{Na}^{+} / \mathrm{H}^{+}\right.$exchanger regulatory factor) gene mutations in human breast cancer. Oncogene 23: 8681-8687, 2004.
20. Shibata T, Chuma M, Kokubu A, Sakamoto M and Hirohashi S: EBP50, a beta-catenin-associating protein, enhances Wnt signaling and is over-expressed in hepatocellular carcinoma. Hepatology 38: 178-186, 2003.

21. Mangia A, Saponaro C, Malfettone A, Bisceglie D, Bellizzi A, Asselti M, Popescu O, Reshkin SJ, Paradiso A and Simone G: Involvement of nuclear NHERF1 in colorectal cancer progression. Oncol Rep 28: 889-894, 2012

22. Malfettone A, Silvestris N, Paradiso A, Mattioli E, Simone G and Mangia A: Overexpression of nuclear NHERF1 in advanced colorectal cancer: Association with hypoxic microenvironment and tumor invasive phenotype. Exp Mol Pathol 92: 296-303, 2012.

23. Gu Y, Yu H, Hao C, Martin TA, Hargest R, He J, Cheng S and Jiang WG: NHERF1 regulates the progression of colorectal cancer through the interplay with VEGFR2 pathway. Oncotarget 8: 7753-7765, 2017.

24. Vaquero J, Nguyen Ho-Bouldoires TH, Claperon A and Fouassier L: Role of the PDZ-scaffold protein NHERF1/EBP50 in cancer biology: From signaling regulation to clinical relevance. Oncogene 36: 3067-3079, 2017.

25. Song GJ, Leslie KL, Barrick S, Mamonova T, Fitzpatrick JM, Drombosky KW, Peyser N, Wang B, Pellegrini M, Bauer PM, et al: Phosphorylation of ezrin-radixin-moesin-binding phosphoprotein 50 (EBP50) by Akt promotes stability and mitogenic function of S-phase kinase-associated protein-2 (Skp2). J Biol Chem 290: 2879-2887, 2015.

26. He J, Lau AG, Yaffe MB and Hall RA: Phosphorylation and cell cycle-dependent regulation of $\mathrm{Na}^{+} / \mathrm{H}^{+}$exchanger regulatory factor-1 by Cdc2 kinase. J Biol Chem 276: 41559-41565, 2001.

27. Peng Z, Wang Q, Zhang Y, He J and Zheng J: EBP50 interacts with EGFR and regulates EGFR signaling to affect the prognosis of cervical cancer patients. Int J Oncol 49: 1737-1745, 2016.

28. Ji MY, Fan DK, Lv XG, Peng XL, Lei XF and Dong WG: The detection of EBP50 expression using quantum dot immunohistochemistry in pancreatic cancer tissue and down-regulated EBP50 effect on PC-2 cells. J Mol Histol 43: 517-526, 2012.

29. Smith PM, Cowan A, Milgram SL and White BA: Tissue-specific regulation by estrogen of ezrin and ezrin/radixin/moesin-binding protein 50. Endocrine 22: 119-126, 2003.

30. Fouassier L, Rosenberg P, Mergey M, Saubaméa B, Clapéron A, Kinnman N, Chignard N, Jacobsson-Ekman G, Strandvik B, Rey C, et al: Ezrin-radixin-moesin-binding phosphoprotein (EBP50), an estrogen-inducible scaffold protein, contributes to biliary epithelial cell proliferation. Am J Pathol 174: 869-880, 2009.

31. Ediger TR, Kraus WL, Weinman EJ and Katzenellenbogen BS: Estrogen receptor regulation of the $\mathrm{Na}^{+} / \mathrm{H}^{+}$exchange regulatory factor. Endocrinology 140: 2976-2982, 1999.

32. Harrington WR, Sheng S, Barnett DH, Petz LN, Katzenellenbogen JA and Katzenellenbogen BS: Activities of estrogen receptor alpha- and beta-selective ligands at diverse estrogen responsive gene sites mediating transactivation or transrepression. Mol Cell Endocrinol 206: 13-22, 2003.

33. Stemmer-Rachamimov AO, Wiederhold T, Nielsen GP, James M, Pinney-Michalowski D, Roy JE, Cohen WA, Ramesh V and Louis DN: NHE-RF, a merlin-interacting protein, is primarily expressed in luminal epithelia, proliferative endometrium, and estrogen receptor-positive breast carcinomas. Am J Pathol 158: 57-62, 2001.

34. Diala ES and Hoffman RM: Hypomethylation of HeLa cell DNA and the absence of 5-methylcytosine in SV40 and adenovirus (type 2) DNA: Analysis by HPLC. Biochem Biophys Res Commun 107: 19-26, 1982.

35. Mudbhary R, Hoshida Y, Chernyavskaya Y, Jacob V, Villanueva A, Fiel MI, Chen X, Kojima K, Thung S, Bronson RT, et al: UHRF1 overexpression drives DNA hypomethylation and hepatocellular carcinoma. Cancer Cell 25: 196-209, 2014.

36. Costello JF, Fruhwald MC, Smiraglia DJ, Rush LJ, Robertson GP, Gao X, Wright FA, Feramisco JD, Peltomäki P, Lang JC, et al: Aberrant CpG-island methylation has non-random and tumour-type-specific patterns. Nat Genet 24: 132-138, 2000.

37. Hoffman RM: Altered methionine metabolism, DNA methylation and oncogene expression in carcinogenesis. A review and synthesis. Biochim Biophys Acta 738: 49-87, 1984.

38. Lao VV and Grady WM: Epigenetics and colorectal cancer. Nat Rev Gastroenterol Hepatol 8: 686-700, 2011. 